\title{
An Improved JPEG Image Compression Technique based on Selective Quantization
}

\author{
Mahmud Hasan \\ Lecturer \\ Department of Allied Engineering \\ Bangladesh University of Textiles
}

\author{
Kamruddin Md. Nur \\ Assistant Professor \\ Dept. of Computer Science \& Engineering \\ Stamford University Bangladesh
}

\author{
Hasib Bin Shakur \\ Lecturer \\ Dept. of Computer Science \& Engineering \\ Stamford University Bangladesh
}

\begin{abstract}
In today's communicative and multimedia computing world, JPEG images play a vast consequential role. The JPEG images have been able to satisfy the users by fulfilling their demand of preserving numerous digital images within considerably less storage space. Although the JPEG standard offers four different sorts of compression mechanism, among them the Baseline JPEG or Lossy Sequential DCT Mode of JPEG is most popular since it can store a digital image by temporarily removing its psychovisual redundancy and thereby offering a very less storage space for a large image. Again, the computational complexity of Baseline JPEG is also considerably less as compression takes place in Discrete Cosine Transform domain. Therefore, Baseline JPEG is substantially useful while storing, sharing and transmitting digital images. Despite removing a large amount of psychovisual redundancy, the Baseline JPEG still contains redundant data in DCT domain. This paper explores the fact and introduces an improved technique that modifies the Baseline JPEG algorithm. It describes a way to further compress a JPEG image without any additional loss while achieving a better compression ratio than that is achievable by Baseline JPEG. The contribution of this work is to incorporate a simple mathematical series with Baseline JPEG before applying optimal encoding and perform a Selective Quantization that essentially does not loss any information after decompression but reduces the redundant data in DCT domain. The proposed technique is tested on over 200 textbook images that are extensively used for testing standard Image Processing and Computer Vision algorithms. The experimental results show that our proposed approach achieves $2.15 \%$ and $14.10 \%$ better compression ratio than that is achieved by Baseline JPEG on an average for gray-scale and true-color images respectively.
\end{abstract}

\section{General Terms:}

JPEG, Huffman Coding

\section{Keywords:}

Baseline JPEG, Compression Ratio, Discrete Cosine Transform, Peak Signal to Noise Ratio, Selective Quantization, $2^{n}$ Based Quantization

\section{INTRODUCTION}

The JPEG has four distinct modes of operation [1, 2, 3, 4, 5, 6]. The Lossy Sequential DCT Based Mode (or Baseline JPEG) is most popular among them since it can achieve highest compression ratio beside preserving maximum possible image quality. Although the expanded lossy mode can achieve more compression, it has not been generalized to the end users since the amount of data loss is much higher. Because the achievable compression ratio is lower, the lossless mode is also not in consideration. Therefore, the Baseline JPEG stands as a standard for compressing digital images [1, 3].

Generally, a digital image contains three distinguished types of redundancy; namely, Inter-Pixel Redundancy, Coding Redundancy and Psychovisual Redundancy [3, 7]. Since a digital image contains a huge amount of psychovisually redundant data, Baseline JPEG focused on removing this redundancy [1]. To achieve the required target, Baseline JPEG follows the steps as illustrated in figure 1. For color images, the color planes are separated before the algorithm is applied and the RGB color domain is transformed to $Y C_{b} C_{r}$ color domain in order to separate the luminance and chrominance signals [7]. The separation of color space is mandatory for color images, but transformation to $Y C_{b} C_{r}$ color domain in addition has been kept optional [8, 9].

The Baseline JPEG uses a default Huffman Coding Table in Entropy Encoding stage so that the encoded bit stream can be uniquely decoded [3]. However, Run-length or Arithmetic Coding can also be applied in this stage without compromising the resulting number of bits since all of them are optimal coding [1, 7]. As an already optimally compressed bit stream can not further be compressed [6, 10], any effort to further compression of a JPEG coded bit stream is meaningless. However, since the Baseline JPEG Default Huffman Coding results different depending on the distribution of the frequency of the input data set, if the data set of an image applied for Huffman Coding is modified, obtaining more optimum result is still possible.

In this paper, our study explores the redundancy of data in DCT domain and reveals a straight forward way to make a further compression of Baseline JPEG without any additional loss of image information. We incorporate a Selective Quantization approach with the Baseline JPEG that essentially reduces a 


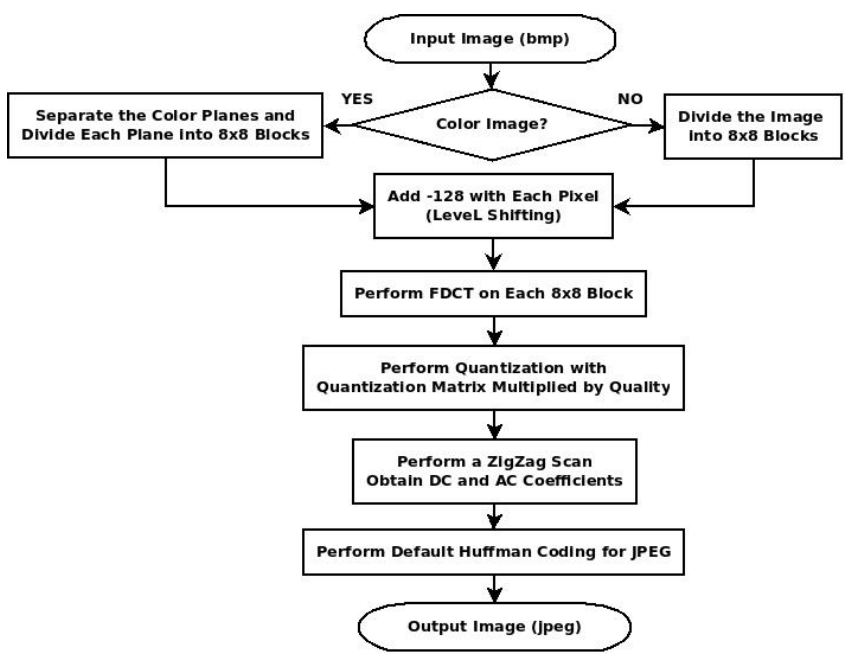

Fig. 1. Flowchart of Baseline JPEG.

particular type of redundancy from DCT domain. Our proposed improvement has been able to obtain better compression ratio or lesser number of bits as compared to Baseline JPEG standard.

The rest of the paper is organized as follows. After a careful explanation of the background of the study, the suggested Selective Quantization Approach is discussed in section 3. For more clarification, a detailed example is worked out in section 4 . The anomalies introduced by our proposed modification are taken into consideration and techniques to handle them efficiently are presented in section 5. Further lossless compression is proved by Comparative Compression Ratio and Peak Signal to Noise Ratio analysis in section 6. In that section, an exact matching of PSNR of our method with the PSNR of Baseline JPEG proves that the image information other than that are lost by Baseline JPEG algorithm is indeed preserved in a lossless manner. Finally, we discuss the application of proposed improvement for convenience of future research work in section 7 and conclude thereafter.

\section{BACKGROUND AND RELATED STUDY}

The motivation of further compression of Baseline JPEG is influenced by the fact that this format is in widespread use in almost all sorts of digital camera and other digital devices. Yet, there have been very few researches that attempted to reduce the number of bits obtained by Baseline JPEG images while keeping the image quality unaffected.

The further studies of JPEG images can be categorized in two branches- one emphasizes the quality enhancement of JPEG images while the other took the further compression into consideration. Being a lossy mode of image compression, JPEG sometimes losses important image information along with psychovisually redundant data. Again, the quality-compression trade-off of digital images persuaded the researchers to improve or measure the quality of well compressed JPEG images [11, 12, 13, 14, 15, 16].

Another branch of studies focused on further compression of JPEG images rather than the quality improvement [9] 17, [18, 19, 20, 21]. Some of these studies [18, 19, 20] approached a lossless manner for further compression as the proposed method does while the other tried to make further compression in a lossy manner. One research attempted to achieve better compression ratio along with better quality [9]
Table 1. JPEG Coefficient Coding Categories.

\begin{tabular}{|c|c|c|}
\hline Range & $\begin{array}{c}\text { DC } \\
\text { Difference } \\
\text { Category }\end{array}$ & AC Category \\
\hline 0 & 0 & N/A \\
\hline$-1,1$ & 1 & 1 \\
\hline$-3,-2,2,3$ & 2 & 2 \\
\hline$-7, \ldots,-\mathbf{4}, \mathbf{4}, \ldots 7$ & 3 & 3 \\
\hline$-15, \ldots,-\mathbf{8}, \mathbf{8}, \ldots, 15$ & 4 & 4 \\
\hline$-31, \ldots-\mathbf{- 1 6}, \mathbf{1 6}, \ldots, 31$ & 5 & 5 \\
\hline$-63, \ldots,-\mathbf{3 2}, \mathbf{3 2}, \ldots, 63$ & 6 & 6 \\
\hline$-127, \ldots,-\mathbf{6 4}, \mathbf{6 4}, \ldots, 127$ & 7 & 7 \\
\hline$-255, \ldots,-\mathbf{1 2 8}, \mathbf{1 2 8}, \ldots, 255$ & 8 & 8 \\
\hline$-511, \ldots,-\mathbf{2 5 6} 6,256, \ldots, 511$ & 9 & 9 \\
\hline$-1023, \ldots,-\mathbf{5 1 2 , 5 1 2}, \ldots, 1023$ & A & A \\
\hline$-2047, \ldots,-\mathbf{1 0 2 4}, \mathbf{1 0 2 4}, \ldots, 2047$ & B & B \\
\hline$-4095, \ldots,-\mathbf{2 0 4 8 , 2 0 4 8}, \ldots, 4095$ & $\mathrm{C}$ & $\mathrm{C}$ \\
\hline$-8191, \ldots,-\mathbf{4 0 9 6}, \mathbf{4 0 9 6}, \ldots, 8191$ & $\mathrm{D}$ & $\mathrm{D}$ \\
\hline$-16383, \ldots,-\mathbf{8 1 9 2 , 8 1 9 2}, \ldots,-16383$ & $\mathrm{E}$ & $\mathrm{E}$ \\
\hline$-32767, \ldots,-\mathbf{1 6 3 8 4 , 1 6 3 8 4}, \ldots, 32767$ & $\mathrm{~F}$ & N/A \\
\hline
\end{tabular}

although it changes Baseline JPEG algorithm considerably. Since JPEG itself defines a variable quantization [2, 3, 4, 7], more compressing a JPEG file in a lossy manner is of no use. Hence, only further compression of JPEG images in a lossless manner deserves attention. Our proposed method, belonging to this category of studies, achieves better compression ratio as compared to JPEG without any additional loss of information. In addition, our proposed Selective Quantization approach is directly applicable to any further compression of JPEG images as referenced in this paper.

\section{SELECTIVE QUANTIZATION APPROACH}

Simply stated, the idea behind our technique is to perform a search on the sequence of DC or AC coefficients [3] obtained by performing a zigzag scan over an $8 \times 8$ two dimensional Discrete Cosine Transformed block of a digital image and to look for coefficients that belong to $\pm 2^{n}$, where $n \geq 1$. That is, we just care for the coefficients $\pm 2, \pm 4, \pm 8, \pm 16, \pm 32, \pm 64 \ldots$. etc. for our proposed technique. We define the coefficients of $\pm 2^{n}$, where $n \geq 1$, as our Candidate Coefficients. If a Candidate Coefficient is encountered, the coefficient is divided by 2 . We term this division as Selective Quantization or $2^{n}$ Based Quantization. The selectively quantized data set is then encoded using the default JPEG Huffman encoding table.

Generally, the Baseline JPEG, as described in figure 1, first divides an image into a number of $8 \times 8$ non-overlapping blocks and then performs a scaling upon them. A $2 D$ Discrete Cosine Transform is then applied on each $8 \times 8$ block. After that, the blocks are ready for quantization by the default JPEG quantization matrix. Performing a zigzag scan over the rounded quantized block results in a sequence of integers. The sequence of integers contain three different types of values- DC Coefficient (first value of each block), AC Coefficient (all values except the first and last value of each block) and a block ending indicator EOB (End of Block). Using the JPEG Coefficient Coding Category Table as shown in Table 1., the coefficients are then categorized for coding. For a particular Differenced DC Coefficient, the Entropy Encoding is performed using The JPEG Default DC Coding Table as given in Table 2. Similar coding table for AC coefficients is also provided by JPEG standard [3].

It should be observed from table 2 that the less the category, the less the entire bit-length of the coded coefficient. For 
Table 2. JPEG Default DC Coding.

\begin{tabular}{|c|c|c|c|c|c|}
\hline Category & Base Code & Length & Category & Base Code & Length \\
\hline 0 & 010 & 3 & 6 & 1110 & 10 \\
\hline 1 & 011 & 4 & 7 & 11110 & 12 \\
\hline 2 & 100 & 5 & 8 & 111110 & 14 \\
\hline 3 & 00 & 5 & 9 & 1111110 & 16 \\
\hline 4 & 101 & 7 & A & 11111110 & 18 \\
\hline 5 & 110 & 8 & B & 111111110 & 20 \\
\hline
\end{tabular}

example, category 6 requires 10 bits to encode a coefficient while category 5 requires only 8 bits. Hence, reducing the category for DC or AC Coefficients must result in reduced number of bits. Again, from table 1, it is seen that only the lower bound conditions (indicated by bold letters) can be further quantized by a factor of 2 since each lower bound value belongs to $\pm 2^{n}$, where $n \geq 1$. As dividing a $2^{n}$ value by 2 must always result in another $2^{n}$ value, we can distinguish our Candidate Coefficients uniquely during decoding phase. Compression by Selective Quantization is lossless since no fractional data will be introduced. In addition, dividing a Candidate Coefficient by 2 reduces its category by 1 , therefore, the final encoded bit stream must be smaller. For example, dividing 512 (category $A$ ) by 2 will result in 256 (category 9). Essentially, category 9 requires less bits ( 16 bits) than that of category $A$ (18 bits). Thus, if we would encode a coefficient having numeric value 512 by Baseline JPEG, it would have taken 18 bits. However, encoding the same coefficient after Selective Quantization approach, we have to allocate only 16 bits. Moreover, dividing a $2^{n}$ by 2 is simply a shift operation that the processor can easily process in general.

The decoding phase of our proposed improvement is relatively simple. We look only for the Candidate Coefficients and simply multiply them by 2 . Then the Baseline JPEG decoding steps are applied. Figure 2 illustrates the Selective Quantization approach. However one problem that may be arised during decoding phase is mentioned and solved in section 5

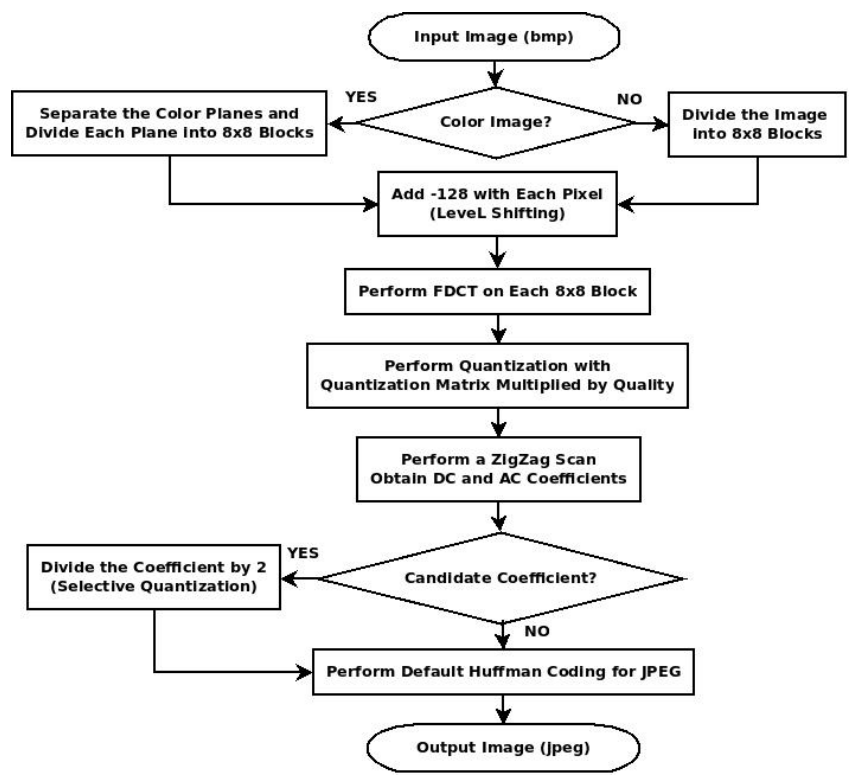

Fig. 2. Flowchart of the Proposed Selective Quantization.

\section{A WORKED OUT EXAMPLE}

Let us consider the sequence of digits $(36,12,0,-1,-4,2, E O B)$ as the coefficients of an $8 \times 8$ block of a digital image after performing zigzag scan over it in figure 3. Let us also consider that the DC coefficient of previous block is 4 . Thus, we have $36-4=32$ for coding the DC value. Since 32 falls in DC difference category 6 , the number of bits required to code it using default Baseline JPEG Huffman Table is 10. But whenever our algorithm is applied, the DC difference 32 is a Candidate Coefficient and hence divided by 2 . Now, 16 falls in DC difference category 5 which requires 8 bits to be encoded. Again, we have a Candidate Coefficient -4 , for which Baseline JPEG requires 6 bits. However, in case of Selective Quantization, it needs only 4 bits to be encoded. Similarly, 2 is a Candidate Coefficient, for which we can reduce 1 bit. Therefore, only from a single block, we could have reduced more $2+2+1=5$ bits that were not under the consideration of Baseline JPEG standard. A $512 \times 512$ gray-scale image has 4096 such blocks, it can be imagined how many bits, in total, can be reduced by our approach having no loss of information rather than those defined by Baseline JPEG itself. The detailed statistical results obtained by implementation of our approach are discussed in the section 6 .

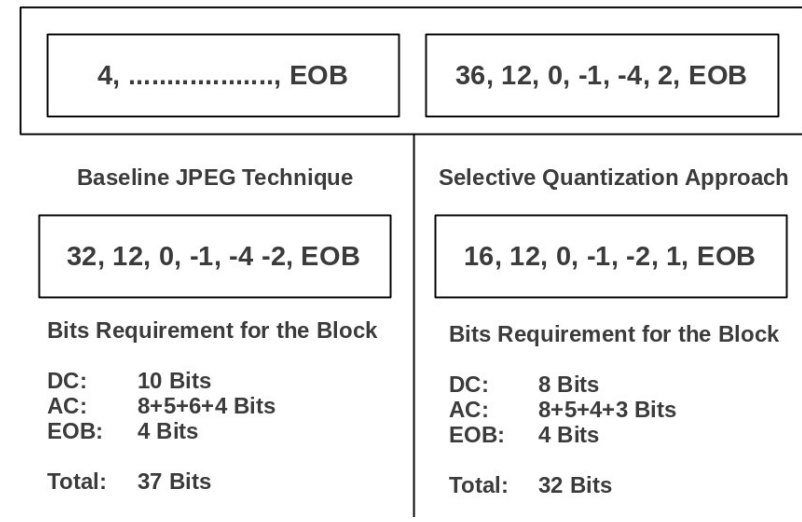

Fig. 3. A Worked Out Example.

\section{HANDLING ANOMALIES}

Problem arises when \pm 2 is encountered as Candidate Coefficient. Since the sequence of DC or AC Coefficients obtained by performing a zigzag scan over an $8 \times 8$ block of a digital image may contain some \pm 1 , it must conflict with \pm 1 obtained as a result of division by 2 of \pm 2 (Selective Quantization). Since the number of symbols to be represented and further distinguished is four in this case, in order to uniquely decode the bit sequence generated by our proposed suggestion simply following Baseline JPEG Decoding Steps is not possible as there will be no distinction between existing \pm 1 and resulting \pm 1 . However, if we increase the total bit length of category 1 from 4 to 5 , it is possible to uniquely decode the bit stream. The one extra bit will help distinguish between the existing \pm 1 and resulting \pm 1 .

Generally, all JPEG coefficients contain a Base Code or Prefix Code that helps the JPEG Decoder find out the category the coefficient belongs to. If the category is $n$, the next $n$ bits immediately after Prefix Code is considered for the coefficient being decoded. For example, let us consider a bit stream starting with prefix 100. The decoder finds the category 2 for this coefficent in a look up table manner [3]. Also, the decoder understands that the next 2 bits after 100 is to be considered for 
Table 3. Percentage of Candidate Values in case of Gray-Scale Images.

\begin{tabular}{lccccc}
\hline $\begin{array}{l}\text { Image } \\
\text { Name }\end{array}$ & $\begin{array}{l}\text { B=Total } \\
\text { Blocks }\end{array}$ & $\begin{array}{c}\text { A=Candidate P=Percentage } \\
\text { Blocks }\end{array}$ & P/A & P/B \\
from A & & & \\
\hline Lena & 4096 & 1460 & 305.9620 & 20.9563 & 7.4698 \\
Baboon & 4096 & 2997 & 384.9374 & 12.8441 & 9.3978 \\
Peppers & 4096 & 1503 & 365.0628 & 24.2889 & 8.9127 \\
Tiger & 4096 & 2505 & 369.2042 & 14.7387 & 9.0138 \\
\hline
\end{tabular}

Table 4. Percentage of Candidate Values in case of True-Color Images.

\begin{tabular}{llcccc}
\hline $\begin{array}{l}\text { Image } \\
\text { Name }\end{array}$ & $\begin{array}{l}\text { Total } \\
\text { Blocks }\end{array}$ & $\begin{array}{c}\text { \% counted } \\
\text { from R } \\
\text { plane }\end{array}$ & $\begin{array}{c}\text { \% counted } \\
\text { from G } \\
\text { plane }\end{array}$ & $\begin{array}{c}\text { \% counted } \\
\text { from B } \\
\text { plane }\end{array}$ & $\begin{array}{c}\text { Total } \\
\text { \% }\end{array}$ \\
\hline Lena & 12288 & 7.0794 & 4.6185 & 3.5277 & 15.23 \\
Baboon & 12288 & 6.2398 & 6.6224 & 4.3932 & 17.26 \\
Peppers & 12288 & 20.2405 & 8.2182 & 7.9890 & 36.44 \\
Tiger & 12288 & 8.2223 & 7.3066 & 6.8863 & 22.42
\end{tabular}

the coefficient being processed. Now, all possible combinations of 2 bits is 4 and these are $00,01,10$ and 11 respectively. Since the decoder knows from table 1 that there can be at most 4 symbols $(-3,-2,+2,+3)$ in category 2 , it considers the first 2 bits combinations 00 as $-3,01$ as -2 and so on. However, we will use a little bit different approach in order to distinguish our existing and resulting $\pm 1 \mathrm{~s}$.

For our case, ambiguities arise only for the coefficients $\pm 1 \mathrm{~s}$. Therefore, we take only category 2 into account. As stated earlier, we should consider the Total Bit Length of category 2 as 5 instead of 4 . Now, whenever the decoder finds a coefficient starting with 011 (for DC only), it first decides the category as 1 . Now, only for category 1 , it will consider the next 1 bit to find out its subcategory. If the next bit is 0 , it will consider the coefficent as existing \pm 1 else it will consider it as resulting \pm 1 . The final bit denotes the sign of the coefficient. If the final bit is 0 , it is -1 and otherwise it is +1 . The similar approach can be done for AC coefficients as well.

Although, at first glance, it seems that increasing one bit in category 2 will also increase total bit length of the coefficients, but it is not the usual case. It may only happen when there is no or very small number of candidate coefficients present in the image signal. However, signal coefficients vary in amplitude and it is almost impossible to predict for which images the propsed method may not work. In general, there are good number of candidate coefficients present in the image signal as studied in 6 and the total number of bits reduced is much greater than the number of times we increase one bit for category 1 .

\section{PERFORMANCE ANALYSIS}

A simulation over 200 textbook images has been conducted for evaluation of performance of our proposed Selective Quantization approach. Since the proposed method depends largely on the frequency of Candidate Coefficients, we searched for the number of Candidate Coefficients appearing in the Selective Quantization step. Around 9.3196\% of coefficients appeared as candidate while taking the gray-scale images into consideration. However, since dividing the color planes substantially increases the probability of Candidate Coefficient occurrence, we obtained $18.2132 \%$ Candidate Coefficients for true-color images. Table 3 and table 4 shows the portion of our study for finding Candidate Coefficients.
Table 5. Comparative Compression Ratio Analysis for Gray-Scale Images.

\begin{tabular}{lcccc}
\hline $\begin{array}{l}\text { Image } \\
\text { Name }\end{array}$ & $\begin{array}{c}\text { Total } \\
\text { Blocks }\end{array}$ & $\begin{array}{c}\text { JPEG } \\
C_{R}\end{array}$ & $\begin{array}{c}\text { Selective } \\
\text { Quantization } \\
C_{R}\end{array}$ & $\begin{array}{c}C_{R} \\
\text { Achieved }\end{array}$ \\
\hline Lena & 4096 & 23.3965 & 24.7145 & 1.3179 \\
Baboon & 4096 & 8.4037 & 9.0055 & 0.6017 \\
Peppers & 4096 & 24.9765 & 26.7238 & 1.7473 \\
Cameraman & 4096 & 17.6763 & 18.7709 & 1.0946 \\
\hline
\end{tabular}

Table 6. Comparative Compression Ratio Analysis for True-Color Images.

\begin{tabular}{lcccc}
\hline $\begin{array}{l}\text { Image } \\
\text { Name }\end{array}$ & $\begin{array}{c}\text { Total } \\
\text { Blocks }\end{array}$ & $\begin{array}{c}\text { JPEG } \\
C_{R}\end{array}$ & $\begin{array}{c}\text { Selective } \\
\text { Quantization } \\
C_{R}\end{array}$ & $\begin{array}{c}C_{R} \\
\text { Achieved }\end{array}$ \\
\hline Lena & 12288 & 14.3466 & 26.4226 & 12.0426 \\
Actress & 12288 & 10.6075 & 15.6040 & 4.9965 \\
Lake & 12288 & 34.0587 & 52.8964 & 18.8377 \\
Tiger & 12288 & 21.1175 & 35.8736 & 14.7561 \\
\hline
\end{tabular}

The application of Selective Quantization over Baseline JPEG results in better compression ratio as described in section 3 . Our simulation reveals the comparison of compression ratio of both Baseline JPEG and Selective Quantization for 200 test images. Although the compression ratio achieved by Selective Quantization in case of gray-scale images is smallonly $2.15 \%$; for true-color images the achievement is $14.10 \%$. This is because the separation of color planes results in higher frequency of Candidate Coefficients as shown in table 5. Table 5 and table 6 shows the portion of comparative compression ratio achieved in Baseline JPEG and Selective Quantization approach for gray-scale and true-color images respectively. Figure 4 and figure 5 show the comparative compression ratio for 75 gray-scale and color images respectively. The term Compression Ratio is defined by the equation 1 .

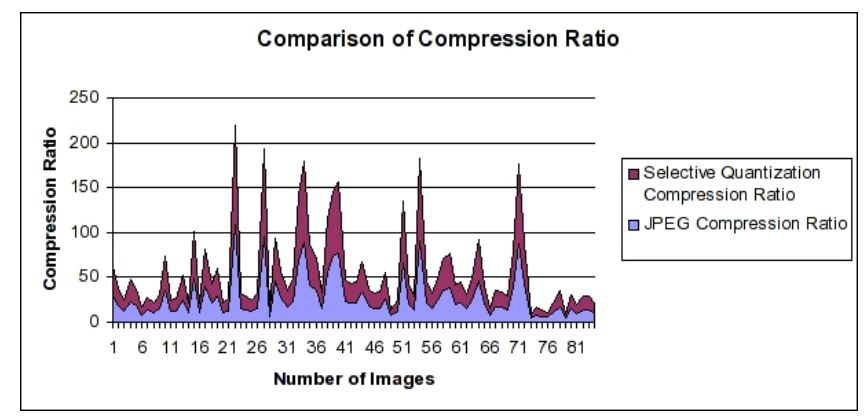

Fig. 4. Comparative Compression Ratio for Gray-Scale Images.

$$
\begin{gathered}
C_{R}=\frac{\text { total }_{\text {inputBits }}}{\text { total }_{\text {outputBits }}} \\
M S E=\frac{1}{m n} \sum_{i=0}^{m-1} \sum_{j=0}^{n-1}[I(i, j)-J(i, j)]^{2} \\
P S N R=10 \log _{10} \frac{M A X_{I}^{2}}{M S E}
\end{gathered}
$$

$\triangle P S N R=P S N R_{J P E G}-P S N R_{\text {SelectiveQuantization }}$ 


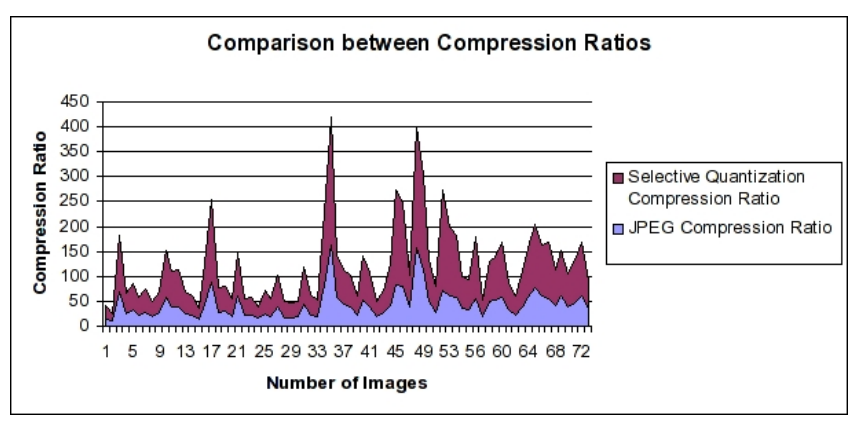

Fig. 5. Comparative Compression Ratio for True-Color Images.

Table 7. Comparison of PSNR Evaluation.

\begin{tabular}{lccc}
\hline $\begin{array}{l}\text { File } \\
\text { Name }\end{array}$ & $P S N R_{J P E G}$ & $P S N R_{\text {SelectiveQuantization }}$ & $\Delta P S N R$ \\
\hline Lena & $37.77 \mathrm{~dB}$ & $37.77 \mathrm{~dB}$ & \\
Baboon & $36.49 \mathrm{~dB}$ & $36.49 \mathrm{~dB}$ & 0.00 \\
Peppers & $37.44 \mathrm{~dB}$ & $37.44 \mathrm{~dB}$ & 0.00 \\
Barbara & $34.97 \mathrm{~dB}$ & $34.97 \mathrm{~dB}$ & 0.00 \\
\end{tabular}

Our demand of no additional loss can be proved by taking the Peak Signal to Noise Ratio into consideration. PSNR is a performance measurement metric extensively used for defining the quality of an image. It is dependent on another performance evaluator Mean Square Error (MSE). MSE and PSNR is defined by equation 2 and 3 respectively. Equation 4 describes the difference between Baseline JPEG PSNR and Selective Quantization PSNR. Since no extra level of noise was introduced due to the application of Selective Quantization approach, no change in $\triangle$ PSNR had been indicated. The $\triangle$ PSNR calculation for some famous test images is given in table 7 .

\section{FURTHER STUDY}

The Selective Quantization approach is an easy, simple and convenient method for further compressing JPEG images with keeping the quality unaffected. This method is directly applicable on the further JPEG image compression techniques as referenced by this paper or on any data compression technique that processes a sequence of integers at least once. The compression ratio achieved by those studies can be enhanced in a lossless manner by incorporating the Selective Quantization approach. The further study of this research may include a detailed study that will help precisely decide what sorts of images may not totally be considered for further compression using the proposed method. The default JPEG quantization matrix can be re-organized following the Selective Quantization approach so that quality assurance is facilitated. Implementation of Selective Quantization with Baseline JPEG can be conducted for specialized computing devices, where storage requirement is a crying need.

\section{CONCLUSION}

In this paper we presented a novel compression technique for further compression of JPEG images without any additional loss of image information. Although the optimal coding stage stands as a barrier while further compressing JPEG images, the application of suggested Selective Quantization approach before optimal coding eased the entire compression scheme. Statistical evidence has been shown against the appearance of Candidate Coefficients and exact matching of PSNR proved the suggested method as lossless. For both gray-scale and true-color images, experimental results clarified that the proposed method gained better compression ratio. Moreover, the future scope of the proposed method has been discussed. The impact of JPEG images is widespread, incorporation of the proposed method with Baseline JPEG will help solve the increasing problem of storing numerous digital images within a little given storage area.

\section{REFERENCES}

[1] Steinmetz, R., Nahrstedt, K.: Multimedia: computing, communications, and applications. Prentice Hall PTR, 1995.

[2] Wallace, G.K.:The JPEG still picture compression standard. IEEE Transactions on Consumer Electronics, 38(1):18-34, February 1992.

[3] Gonzalez, R.C., Woods, R.E.: Digital Image Processing. 2nd ed, p.520.

[4] Pennebaker, W.B., Mitchell, J.L.: JPEG Still Image Data Compression Standard. Van Nostrand Reinhold, New York, 1993.

[5] Miano, J.: Compressed Image File Formats: JPEG, PNG, GIF, XBM, BMP. New York: ACM Press, 1999.

[6] Hankerson, D., Harris, G.A., JohnsonJr., P.D.: Introduction to Information Theory and Data Compression. Boca Raton, FL: CRC, 1997.

[7] Acharya, T., Ray, A.K.: Digital Image Processing: Principles and Applications. John Wiley \& Sons, Inc. ISBN: 10 0-47171998-6, 2005.

[8] Wikipedia: The JPEG Codec Example. http://en.wikipedia.org/wiki/JPEG\# JPEG_codec_example.

[9] Bauschke, H.H., Hamilton, C.H., Macklem, M.S., McMichael, J.S., Swart, N.R.: Recompression of JPEG Images by Requantization. IEEE Transactions on Image Processing, vol. 12, no. 7, July 2003.

[10] Nelson, M., Gailly, J.L.: The Data Compression Book. 2nd ed. New York: M \& T Books, 1996.

[11] Takezawa, M., Sanada, H., Watanabe, K.: Quality Improvement Technique for JPEG Images with Fractal Image Coding. IEEE, 2005.

[12] Richter, T.: Visual quality improvement techniques of HDPhoto/JPEG-XR. 15th IEEE International Conference on Image Processing, 2008.

[13] Gunawan, I.P., Halim, A.: Haar wavelet decomposition based blockiness detector and picture quality assessment method for JPEG images. International Conference on Advanced Computer Science and Information System (ICACSIS), 2011.

[14] Zhou, W., Sheikh, H.R., Bovik, A.C.: No-reference perceptual quality assessment of JPEG compressed images. IEEE International Conference on Image Processing, 2002.

[15] Altous, S., Samee, M.K., Gotze, J.: Reduced reference image quality assessment for JPEG distortion. ELMAR, 2011 Proceedings.

[16] Gastaldo, P., Zunino, R.: No-reference quality assessment of JPEG images by using CBP neural networks. International Symposium on Circuits And Systems (ISCAS), 2004.

[17] Stirner, M., Seelmann, G.: Improved Redundancy Reduction for JPEG Files. Picture Coding Symposium by EURASIP, 2007. ISBN: 978-989-8109-05-7.

[18] Bauermann, I., Steinbach, E.: Further Lossless Compression of JPEG Images. Proc. of Picture Coding Symposium, San Fransisco, USA, Dec 15-17, 2004. 
[19] Matsuda, I., Nomoto, Y., Wakabayashi, K., Itoh, S.: Lossless Re-Encoding of JPEG Images using Block-Adaptive Intra Prediction. 16th European Signal Processing Conference (EUSIPCO 2008), Lausanne, Switzerland, August 2529, 2008 .

[20] Zhong-Hua, Z., Wen-Yan, W.: A lossless compression method of JPEG file based on shuffle algorithm. 2nd Interna- tional Conference on Advanced Computer Control (ICACC), 2010.

[21] Golner, M.A., Mikhael, W.B., Krishnan, V., Ramaswamy A.: Region based variable quantization for JPEG image compression. Proceedings of the 43rd IEEE Midwest Symposium on Circuits and Systems, 2000 